Case Report

\title{
Maternal Bradycardia Associated with Betamethasone Administration During Pregnancy
}

\author{
Mojirayo A. Sarumi, James W. Hole, and Robert B. Gherman \\ Division of Maternal-Fetal Medicine, WellSpan Health, York, PA, USA \\ Correspondence should be addressed to Robert B. Gherman; ghermdoc@gmail.com
}

Received 5 April 2019; Accepted 22 August 2019; Published 16 October 2019

Academic Editor: Giovanni Monni

Copyright (c) 2019 Mojirayo A. Sarumi et al. This is an open access article distributed under the Creative Commons Attribution License, which permits unrestricted use, distribution, and reproduction in any medium, provided the original work is properly cited.

\begin{abstract}
Background. Maternal risks of betamethasone have been rarely reported. Case. At 36 weeks' gestation, a previously healthy 23-year-old gravida with fetal intrauterine growth restriction was admitted to the hospital for steroid administration. Twenty-six hours after the first dose of betamethasone, a maternal bradycardia was initially noted and eventually nadired at 41 beats per minute. Consultation with the cardio-electrophysiology service revealed no other apparent etiologies for the sinus bradycardia. Due to the asymptomatic nature of the maternal bradycardia, pharmacologic interventions were not recommended. With observation alone, a normal maternal heart rate returned by forty-nine hours after the original betamethasone injection. The patient subsequently had an uneventful intrapartum course. Conclusion. Maternal bradycardia can be associated with antenatal betamethasone administration. Due to the transient nature of this side effect, expectant management is recommended as the treatment option for asymptomatic patients.
\end{abstract}

\section{Introduction}

Antenatal corticosteroid administration has been shown to reduce the risk of neonatal intraventricular hemorrhage, necrotizing enterocolitis, and respiratory distress syndrome [1]. Therefore, a single course of corticosteroids is currently recommended for patients at risk of preterm delivery within seven days who are within the gestational age of 24 weeks through 33 weeks and 6 days. Administration of betamethasone to women at risk for late preterm $(34+0-36+6$ weeks) delivery has also been shown to be associated with significant reductions in the incidence of severe respiratory complications, transient tachypnea of the newborn, surfactant use, and bronchopulmonary dysplasia [2]. The Antenatal Steroids for Term Elective Caesarean Section trial similarly found lower rates of admission to neonatal intensive care units for respiratory complications in the betamethasone group [3].

Maternal side effects related to betamethasone administration during pregnancy have been rarely reported. Previously reported complications have included maternal hyperglycemia and hypokalemic periodic paralysis [4]. We searched PUBMED, using the mesh terms, "betamethasone," "bradycardia," "heart rate" "side effects," and "pregnancy," but did not find any previously reported cases of betamethasone associated bradycardia during pregnancy.

\section{Case}

We obtained a waiver from our Institutional Review Board to review this case and patient consent was likewise obtained. A previously healthy 23 -year-old Gravida 2 Para 1, presented at $36+4$ weeks' gestation from an outside hospital for evaluation of severe fetal intra-uterine growth restriction. Her obstetric history was remarkable for an uncomplicated term vaginal delivery. She had no prior history of any cardiac symptoms or diagnosis. The patient denied any exposure to illicit drugs. Her medications included oral prenatal vitamins. Her body mass index was $28.5 \mathrm{~kg} / \mathrm{m}^{2}$ (182 pounds, $\mathrm{k} 66$ inches). She had a normal 1-hour glucose tolerance test of $97 \mathrm{mg} / \mathrm{dl}$. She had received the flu and the tetanus-diphtheria-pertussis vaccines several months prior to her admission. The patient had not received any previous courses of betamethasone during the pregnancy. An initial dose of intramuscular betamethasone had been given eleven hours prior to evaluation at our institution.

The patient's temperature was 97.1 degrees Fahrenheit and her pulse was noted to be 67 beats per minute on admission to our institution. External monitoring showed a reactive fetal 
TABLE 1: Vital signs timeline.

\begin{tabular}{lcccccc}
\hline & Time & Temperature & Pulse & Respiratory rate & Blood pressure & Oxygen saturation \\
\hline & $01 / 12 / 18$ 21:13:00 & $37.4(99.4)$ & 64 & 16 & $118 / 69$ & 95 \\
Hospital admission & $01 / 12 / 1822: 44: 08$ & $36.8(98.2)$ & 67 & 14 & $109 / 71$ & 97 \\
& $01 / 13 / 1805: 58: 43$ & $36.7(98.1)$ & 72 & 14 & $109 / 66$ & 95 \\
& $01 / 13 / 1807: 55: 23$ & $36.7(98.1)$ & 60 & 20 & $112 / 70$ \\
& $01 / 13 / 1814: 35: 45$ & $36.7(98.1)$ & 59 & 16 & $112 / 59$ & 95 \\
2hours, 35 minutes after second & $01 / 13 / 1822: 38: 50$ & $36.4(97.6)$ & 54 & 16 & $117 / 69$ & 95 \\
dose of BMZ & $01 / 14 / 1804: 50: 07$ & $36.7(98.1)$ & 49 & 12 & - & 96 \\
& $01 / 14 / 1805: 07: 21$ & - & 41 & 16 & $95 / 81$ & 96 \\
Hospital discharge & $01 / 14 / 1807: 42: 11$ & $36.4(97.6)$ & 53 & 16 & 96 \\
\hline
\end{tabular}

heart rate tracing and there were no contractions noted on the tocodynamometer. Ultrasound revealed an estimated fetal weight of 1,704 grams (less than first percentile for gestational age), without any apparent fetal anomalies. Middle cerebral artery (peak systolic velocity 1.29 MoM) and umbilical artery Doppler indices (S/D ratio 2.41, PI 0.83) were both within the normal range. Laboratory evaluation included the following: hematocrit $35.4 \%$, platelets $318 \times 10^{9} / \mathrm{L}$, creatinine $0.61 \mathrm{mg} / \mathrm{dl}$, aspartate aminotransferase $11 \mathrm{U} / \mathrm{L}$, alanine aminotransferase $8 \mathrm{U} / \mathrm{L}$, lactate dehydrogenase $111 \mathrm{U} / \mathrm{L}$, uric acid $5.3 \mathrm{mmoL} / \mathrm{L}$, total protein/creatinine ratio 0.14 , thyroid stimulating hormone $0.46 \mathrm{mIU} / \mathrm{L}$, and negative urine drug screen. The serum potassium was $4 \mathrm{mmol} / \mathrm{L}$. Throughout the first day of the inpatient admission, the maternal pulse remained between 60 and 72 beats per minute. Two and a half hours after the administration of the second dose of betamethasone acetate, the maternal pulse dropped to below 60 beats per minute (Table 1). On the second inpatient day, the pulse nadired at 41 beats per minute. An electrocardiogram (Figure 1) revealed sinus bradycardia (46 beats per minute, PR 138s, QRS $86 \mathrm{~s}$, QTC $426 \mathrm{~s}$ ). The patient remained asymptomatic without any complaints of lightheadedness, dizziness, fatigue, dyspnea, or chest pain. Consultation with the cardio-electrophysiology service revealed no other apparent etiologies for the sinus bradycardia. Due to the asymptomatic nature of the maternal bradycardia, pharmacologic interventions were not recommended. With observation alone, a normal maternal heart rate returned by forty-nine hours after the original betamethasone injection. The patient was discharged to home, with a pulse of 67 beats per minute.

At $36+6$ weeks' gestation, the patient readmitted and underwent a primary cesarean delivery due to a nonreassuring fetal heart tracing. The patient delivered a 1755-gram viable female infant. Her pulse rate remained within the normal range during the intrapartum and postpartum periods.

\section{Comment}

Sinus bradycardia is defined as a cardiac rate less than sixty beats per minute and occurs when atrial depolarization is initiated from the sino-atrial node. The most common etiology is simply a resting sinus bradycardia. Other etiologies in the differential diagnosis include hypothyroidism, cardiac ischemia or infarction, hypothermia, hyperkalemia, or autonomic disorders that produce an increase in vagal tone relative to sympathetic tone [5].

To date, there have been isolated case reports in the literature of steroid related bradycardia; all of these have occurred in a nonpregnant population. The largest descriptive study to date included sixty-one children treated with one to five $\mathrm{mg} /$ $\mathrm{kg} /$ day of prednisone [6]. Taylor and Gaco reported a symptomatic sinus bradycardia which was induced by a five-day course of high dose oral prednisolone in a multiple sclerosis patient [7]. Marinov et al. reported a case of sinus bradycardia after a single dose of intravenous dexamethasone for post-operative nausea and vomiting prophylaxis during anesthesia induction [8]. Others have reported sinus bradycardia induced by intravenous methylprednisolone $[9,10]$.

The precise mechanism through which betamethasone causes bradycardia has not been fully established. Animal studies have shown that high-dose corticosteroid therapy may depress the cardiovascular alpha- and beta-adrenergic receptor sensitivity [11]. Other studies have suggested a transient shift in renal electrolyte excretion [12], steroid-induced variations in blood pressure [10], or abnormal levels of serum electrolytes [13]. Puntis et al. has postulated that the preservatives used in the drug preparation may be etiologic [14].

Our patient had an onset of bradycardia approximately 26 hours after the initial dose of betamethasone. Her bradycardia was noted to resolve approximately 23 hours after its initial diagnosis (49hours after initial betamethasone administration). This time frame is in keeping with prior reports in the literature, which describe a time range of 48-96hours of bradycardia onset after initial glucocorticoid administration [6-8]. There have been longer instances of bradycardia onset of 5-7 days after treatment completion [9, 10]. Most of these cases had spontaneous resolution of the bradycardia; medical agents were required in a few instances to ameliorate the bradycardia using intravenous atropine [8], as well as isoproterenol [10].

Our case report possesses limited generalizability, as observational studies with a thorough design and larger patient population are necessary to further evaluate the incidence of betamethasone related maternal bradycardia. Although the package insert for betamethasone describes bradycardia as a potential side effect, we suspect that most obstetricians are not aware of this complication. We propose 


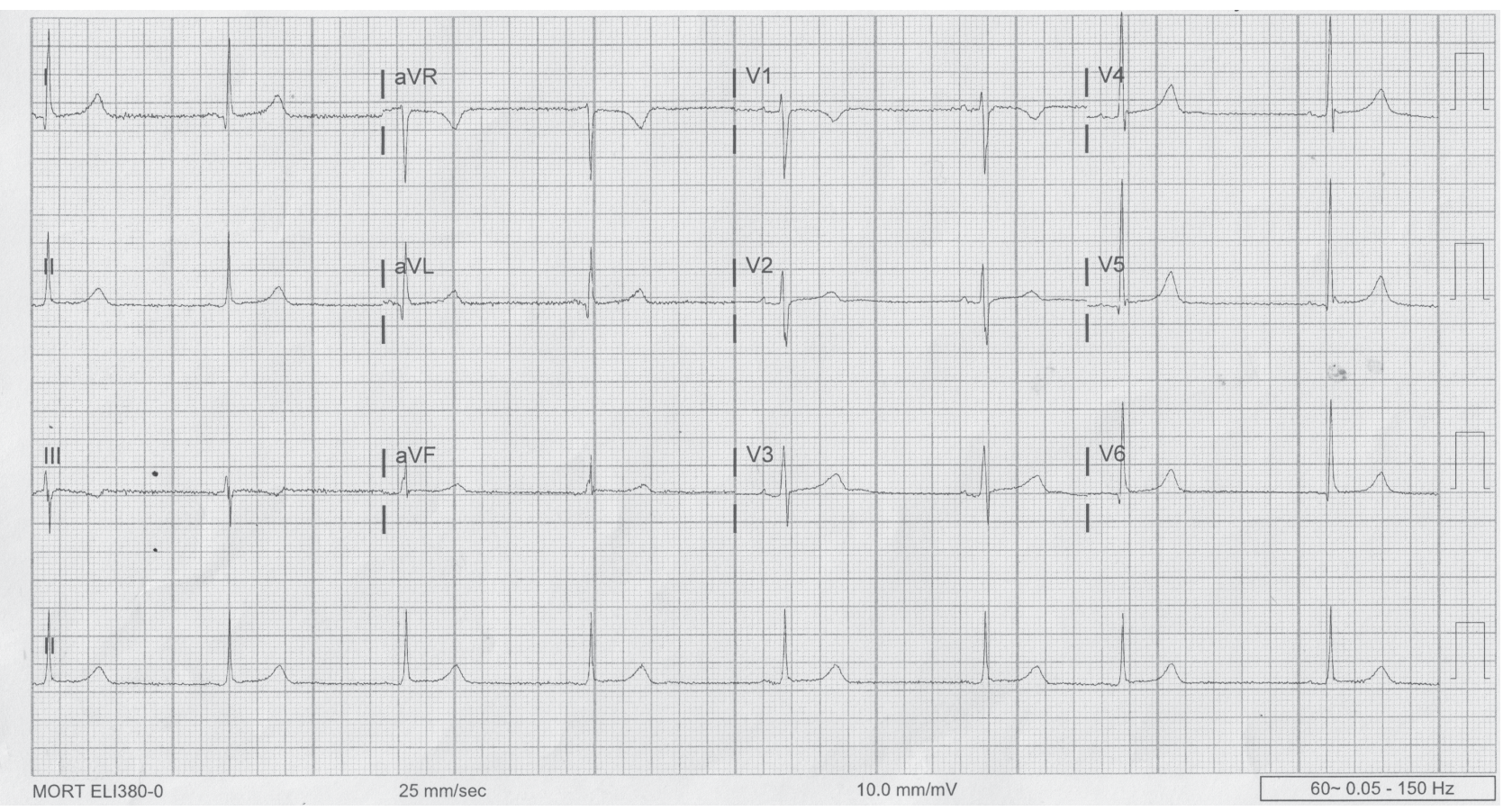

FIGURE 1: Maternal electrocardiogram from second inpatient day.

a consideration of a period of maternal observation and performance of vital signs when corticosteroids are administered in the outpatient setting.

\section{Conflicts of Interest}

The authors declare that they have no conflicts of interest.

\section{References}

[1] “Obstetric Care Consensus No. 4: Periviable Birth," Obstetrics \& Gynecology, vol. 127, no. 6, pp. e157-e169, 2016.

[2] C. Gyamfi-Bannerman, E. A. Thom, S. C. Blackwell et al., "Antenatal betamethasone for women at risk for late preterm delivery. NICHD maternal fetal medicine units network," New England Journal of Medicine, vol. 374, pp. 1311-1320, 2016.

[3] A. M. Nada, M. M. Shafeek, M. A. Maraghy, A. H. El Nageeb, A. S. Salah El Din, and M. H. Awad, "Antenatal corticosteroid administration before elective cesarean section at term to prevent neonatal respiratory morbidity: a randomized controlled trial," European Journal of Obstetrics \& Gynecology and Reproductive Biology, vol. 199, pp. 88-91, 2016.

[4] C. M. Teagarden and C. W. Picardo, "Betamethasone-induced hypokalemic periodic paralysis in pregnancy," Obstetrics \& Gynecology, vol. 117, no. 2, pp. 433-435, 2011.

[5] P. Vijayaraman and K. Ellengogen, "Types of bradyarrhythmia and indication for pacemakers," in Hurst's the Heart Manual of Cardiology, R. Walsh, J. Fang, and V. Fuster, Eds., pp. 136-143, McGraw-Hill Companies, Inc, NY, 12th edition, 2013.

[6] A. van der Gugten, M. Bierings, and J. Frenkel, "Glucocorticoidassociated bradycardia," Journal of Pediatric Hematology Oncology, vol. 30, pp. 172-175, 2008.
[7] M. R. Taylor and D. Gaco, "Symptomatic sinus bradycardia after a treatment course of high dose prednisone," Journal of Emergency Medicine, vol. 45, pp. e55-e58, 2013.

[8] M. Marinov, M. U. Fuessel, and A. F. Unterrainer, "Bradycardia after dexamethasone for postoperative nausea and vomiting prophylaxis during induction of anaesthesia," British Journal of Anaesthesia, vol. 111, pp. 1025-126, 2013.

[9] F. Osuagwu and B. Jahnke, "Intravenous methylprednisoloneinduced nocturnal sinus bradycardia in a multiple sclerosis patient," The Primary Care Companion for CNS Disorders, vol. 18, no. 2, p. 10, 2016.

[10] N. Tvede, L. P. Nielsen, and V. Andersen, "Bradycardia after highdose intravenous methylprednisolone therapy," Scandinavian Journal of Rheumatology, vol. 15, no. 3, pp. 302-304, 1986.

[11] E. Hall, M. Plaster, and J. M. Braughler, "Acute cardiovascular response to a single dose of methylprednisolone and its effects on the response to norepinephrine and isoproterenol," Proceedings of the Society for Experimental Biology and Medicine, vol. 173, no. 3, pp. 338-343, 1983.

[12] S. Fujimoto, H. Kondoh, Y. Yamamoto, S. Hisanaga, and K. Tanaka, "Holter electrocardiogram monitoring in nephritic patients during methylprednisolone pulse therapy," American Journal of Nephrology, vol. 10, no. 3, pp. 231-236, 1990.

[13] A. Al Shibli, I. Al Attrach, and M. A. Hamdan, "Bradycardia following oral corticosteroid use: case report and literature review," Arab Journal of Nephrology Transplant, vol. 5, no. 1, pp. 47-49, 2012.

[14] J. W. L. Puntis, M. E. I. Morgan, and G. M. Durbin, "Dexamethasone-induced bradycardia," The Lancet, vol. 2, no. 8624 , p. $1372,1988$. 


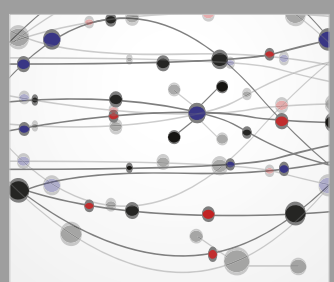

The Scientific World Journal
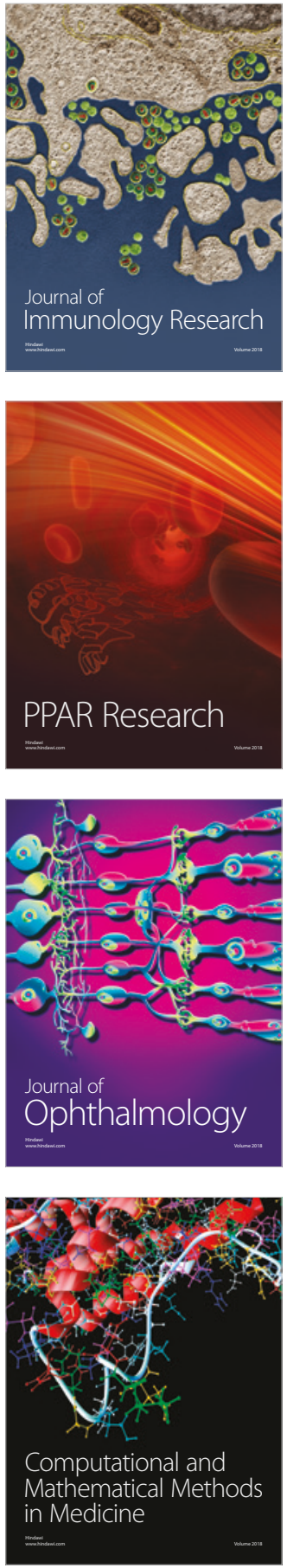

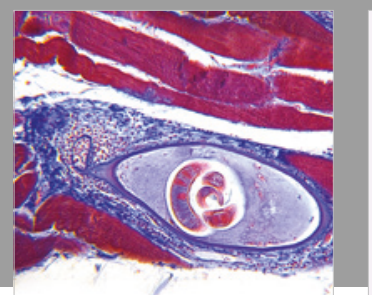

Gastroenterology Research and Practice

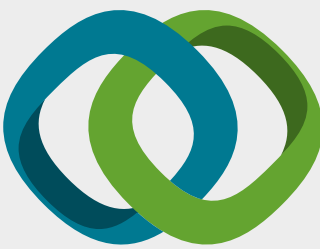

\section{Hindawi}

Submit your manuscripts at

www.hindawi.com
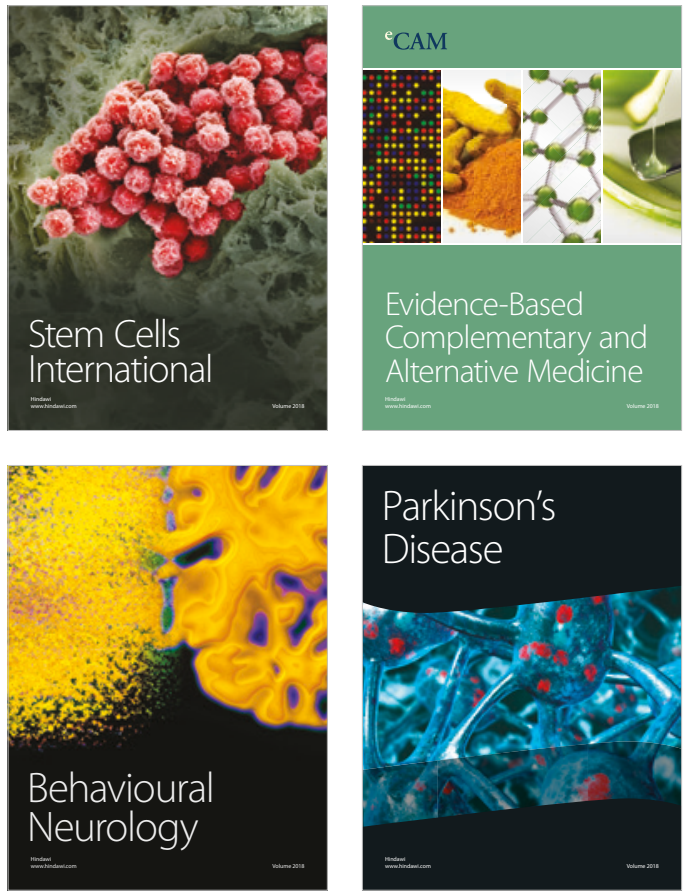

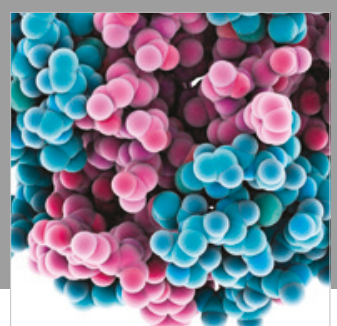

ournal of

Diabetes Research

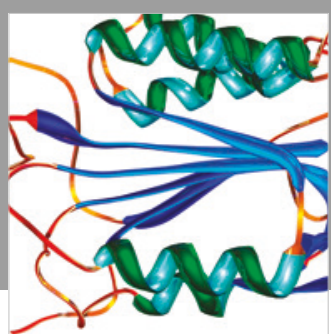

Disease Markers
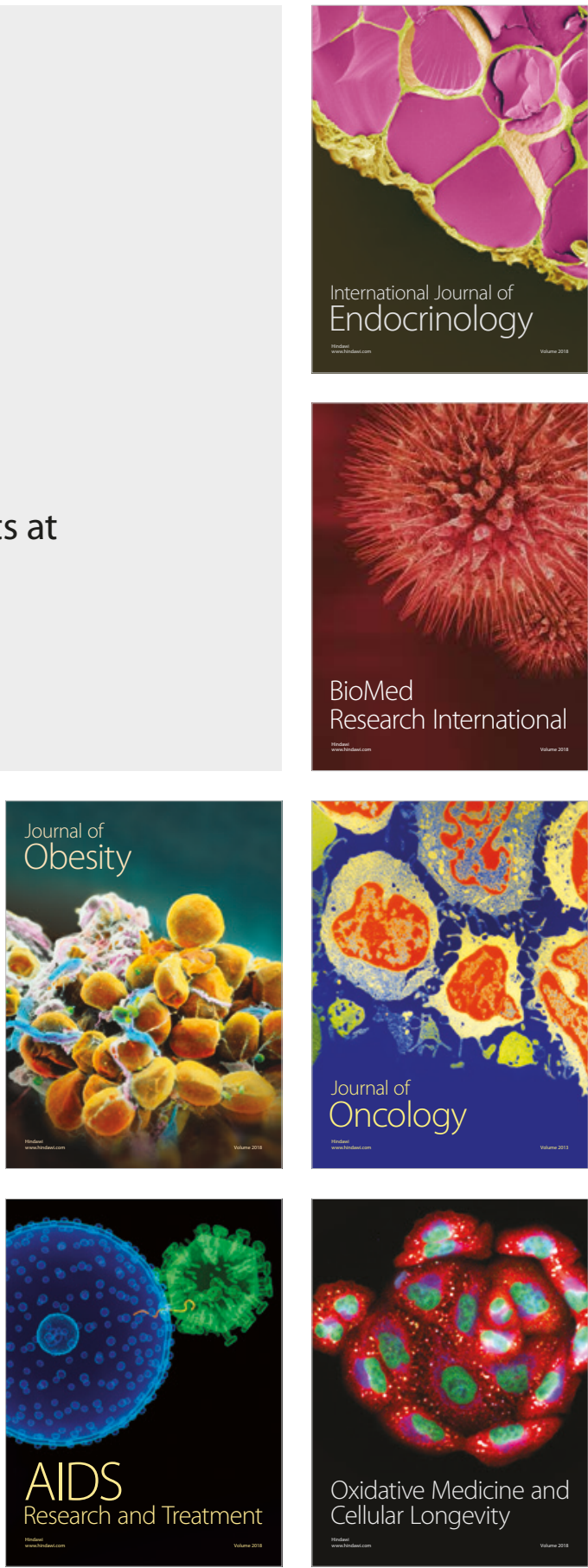